\title{
On $J^{*}-$ Class of $\Pi^{*}-$ Regular Semigroups \\ Xiaoqiang LUO \\ Department of mathematics Sichuan University of Arts and Science, Dazhou, Sichuan, 635000 , China
}

Keywords $\Pi^{\star}$-regular semigroup, Completely $\Pi^{*}$-regular semigroups, $J^{\star}$-class, Idempotent

Abstracts In the paper, we define the equivalence relations on $\Pi^{*}$-regulur semigroups, to show $J^{*}$-class contains an idempotent with some characterizations.

\section{Introduction}

According to [1] some good results, in this paper we consider $J^{*}$-class of $\Pi^{*}$-regular semigroups and completely $\Pi^{*}$-regular semigroups.(see[2])

Remark The marks we don't illustrate in this paper please see reference ([3], [4],[5]).

Let $S$ be a $\Pi^{*}$-regular semigroup.Define on $S$ the equivalence relations $L^{*}, R^{*}, H^{*}, J^{*}$ by

$$
\begin{aligned}
&(a, b) L^{*}(x, y) \Leftrightarrow S(a, b)^{m}=S(x, y)^{n} \\
&(a, b) R^{*}(x, y) \Leftrightarrow(a, b)^{m} S=(x, y)^{n} S \\
& H^{*} \Leftrightarrow L^{*} \mathrm{I} R^{*} \\
&(a, b) J^{*}(x, y) \Leftrightarrow S(a, b)^{m} S=S(x, y)^{n} S \text { (see[3]) }
\end{aligned}
$$

where $m, n$ are the smallest positive integers such that $(a, b)^{m},(x, y)^{n}$ are regulare, i.e. $(a, b)^{m},(x, y)^{n} \in S$. In what follows we will denote by

$$
L_{(a, b)}^{*}\left(R_{(a, b)}^{*}, H_{(a, b)}^{*}, J_{(a, b)}^{*}\right)
$$

the $L^{*}-, R^{*}-, H^{*}-, J^{*}$-class containing an element $(e, e)$ of $S$. According to the hypothesis, we had drawn the following conclusions. (see[1])

Lemma 1. Let $S$ be a $\Pi^{*}$-regular semigroup.Then each idempotent $(e, e)$ of $S$ is a right(left,twosided) identity for regular elements from

$$
L_{(e, e)}^{*}\left(R_{(e, e)}^{*}, J_{(e, e)}^{*}\right)
$$

Lemma 2. In a $\Pi^{*}$-regular semigroup $S$ every $H^{*}$-class contains at most one idempotent.

Lemma 3. Let $S$ be a $\Pi^{*}$-regular semigroup, $(a, b) \in S$ and $p$ the the smallest positive integers such that $(a, b)^{p} \in S$. Then

$$
(a, b)^{p} \in L_{(a, b)}^{*} \cap R_{(a, b)}^{*}=H_{(a, b)}^{*} .
$$

Lemma 4. Let $S$ be a $\Pi^{*}$-regular semigroup. Then

(1) every $J^{*}$-class contains at least one idempotent;

(2) $G_{(e, e)} \subseteq H_{(e, e)} \subseteq J_{(e, e)}$ for every $e \in E$.

Lemma 5. Let $S$ be a $\Pi^{*}$-regular semigroup. Then (for some $(e, e),(f, f) \in E$ )

$$
J_{(e, e)}^{*}=J_{(f, f)}^{*},(e, e)(f, f)=(f, f)(e, e)=(f, f) \Rightarrow(e, e)=(f, f) \text {. }
$$


Lemma 6 . Let $S$ be a $\Pi^{*}$-regular semigroup. Then for some $(u, v) \in S,(e, e) \in E$,

$$
J_{(e, e)}^{*}=J_{(e, e)(u, v)(e, e)}^{*} \Longrightarrow(e, e)(u, v)(e, e) \in G_{(e, e)} \text {. }
$$

Theorem 1. Let $(e, e)$ be an idemptent of a $\Pi^{*}$-regular semigroup $S$. Then $G_{(e, e)} \subseteq H_{(e, e)}^{*}$, furthermore, if $(u, v) \in H_{(e, e)}^{*}$ and $p$ is the smallest positive integer such that $(u, v)^{P} \in S$, then $(u, v)^{q} \in G_{(e, e)}$ for every $q \geq p$.

Theorem 2. Let $S$ be a $\Pi^{*}$-regular semigroup. Then (for some $(a, b),(x, y) \in S$ )

$J^{*}$-class plays a very active role in

$$
J_{(a, b)(x, y)}^{*}=J_{(x, y)(a, b)}^{*} \text {. }
$$

$\Pi^{*}$-regular semigroups, since the emergence of Idempotents. Here we get some good results.

\section{Main Results}

Let $V$ be the set of all inverse elements of $S$ ([3]), $E$ is the set of all idempotent of $S$.

Lemma 7 . Let $S$ be a $\Pi^{*}$-regular semigroup.Then for some $\left.(e, e),(f, f) \in E\right)$

$$
J_{(e, e)}^{*}=J_{(f, f)}^{*}=J_{(e, e)(f, f)}^{*}=J_{(e, e)}^{*} \text {. }
$$

Proof. Let $S(e, e) S=S(f, f) S \cdot$ 'Then

$$
(e, e)=(a, b)(f, f)(e, e)=(a, b)(f, f)(u, v)(e, e)((a, b),(u, v) \in S)
$$

Whence by theorem 2 we have

$$
\begin{aligned}
& J_{(f, f)}^{*}=J_{(e, e)}^{*}=J_{(a, b)(f, f)(u, v)(e, e)}^{*}=J_{((a, b)(f, f))((f, f)(u, v)(e, e))}^{*} \\
& =J_{(f, f)(u, v)(e, e)(a, b)(f, f)}^{*}
\end{aligned}
$$

And since $(f, f)(u, v)(e, e)(a, b)(f, f) \in E$ by lemma 5 we attain that $\mathrm{E}$

and

$$
\begin{aligned}
& (f, f)=(f, f)(u, v)(e, e)(a, b)(f, f) \\
& (f, f)(u, v)(f, f) \in G_{(f, f)} .
\end{aligned}
$$

Analogously

$$
(f, f)(a, b)(f, f) \in G_{(f, f)} \text { So }
$$

$$
((f, f)(e, e)(f, f))^{n}=((f, f)(a, b)(f, f)(f, f)(u, v)(f, f))^{p} \in G_{(f, f)} \subseteq J_{(f, f)}^{*}
$$

for every $n, p \in Z^{+}$. Thus

$$
S(f, f) S=S((f, f)(e, e)(f, f))^{p} S \subseteq S((e, e)(f, f))^{p} S .
$$

and it's opposite inclusion also holds we have

Hence

$$
S(f, f) S=S((e, e)(f, f))^{p} S .
$$

$$
J_{(e, e)}^{*}=J_{(f, f)}^{*}=J_{(e, e)(f, f)}^{*}=J_{(e, e)}^{*} .
$$


Theorem 3. Let $S$ be a $\Pi^{*}$-regular semigroup. Then (for some $(a, b),(x, y) \in S$ )

$$
\mathcal{S}_{(a, b)(x, y)}^{* *}=\mathcal{S}_{(e, e)(f, f)}^{*} \cdot\left(m, n \in Z^{+}\right)
$$

Proof. Let $r$ be the the smallest positive integers such that $((a, b)(x, y))^{r} \in \operatorname{Re} g S$.' Then

$$
\begin{array}{r}
((a, b)(x, y))^{r} \in J_{((a, b)(x, y))}^{*},((a, b)(x, y))^{r} \in G_{(h, h)} \subseteq J_{(h, h)}^{*}, \\
(h, h)(a, b)(x, y)=(a, b)(x, y)(h, h) \in G_{(h, h)},((h, h) \in E)
\end{array}
$$

Now we prove by induction on $p$ that

$$
(h, h)(a, b)^{p}(h, h) \in G_{(h, h)},(h, h)(x, y)^{p}(h, h) \in G_{(h, h)}
$$

for every $p \geq 0$. Suppose $(h, h)(a, b)^{p}(h, h) \in G_{(h, h)}(p \geq 0)$, Then

$$
\left((h, h)(a, b)^{p}(h, h)\right)(h, h)(a, b)(x, y)=(h, h)(a, b)^{p+1}(x, y)(h, h) \in G_{(h, h)} .
$$

Let $(u, v)$ be an inverse element of

Then

$$
(h, h)(a, b)^{p+1}(x, y)(h, h) \text { in } G_{(h, h)},
$$

$$
(h, h)=(h, h)(a, b)^{p+1}(x, y)(u, v)(h, h),
$$

Whence we have that $(h, h)(a, b)^{p+1}$ is regulare.Hence by theorem 2

$$
J_{(h, h)}^{*}=J_{(h, h)(a, b)^{p+1}}^{*}=J_{(h, h)(a, b)^{p+1}(h, h)}^{*} .
$$

Now by lemma $6(h, h)(a, b)^{p+1}(h, h) \in G_{(h, h)}$ and since $(h, h)(a, b)^{0}(h, h) \in G_{(h, h)}$ the first part is proved.In a similar way we can show the second part .

Since $(a, b)^{m} \in G_{(e, e)}$ by the first part of (2) we get

$$
(h, h)(e, e)(a, b)^{m}(h, h) \in G_{(h, h)} .
$$

Let $(u, v)$ be an inverse element of $(h, h)(e, e)(a, b)^{m}(h, h)$ in $G_{(h, h)}$, Then $(h, h)=(h, h)(e, e)(a, b)^{m}(u, v)(h, h),(h, h)(e, e)_{\text {is regular. }}$

Thus

So

$$
J_{(h, h)}^{*}=J_{(h, h)(e, e)}^{*}=J_{(h, h)(e, e)(h, h)}^{*}
$$

$$
(h, h)(e, e)(h, h) \in G_{(h, h)}
$$

and analogously

$$
(h, h)(f, f)(h, h) \in G_{(h, h)} .
$$

Hence

$$
(h, h)(e, e)(h, h),(h, h)(f, f)(h, h) \in G_{(h, h)}
$$

Now we show

$$
(h, h)((e, e)(f, f))^{p}(h, h) \in G_{(h, h)}
$$

for every $p \geq 0$.

From

$$
(h, h)(f, f)(h, h) \in G_{(h, h)} \text { we attain }
$$

$$
(h, h)=(h, h)(e, e)(u, v)(f, f)(h, h)=(h, h)(f, f)(h, h)(e, e)(s, t) \text {, }
$$


Here $(f, f)(h, h)(e, e)$ and $(e, e)(f, f)(h, h)$ are regular And $J_{(h, h)}^{*}=J_{(e, e)(f, f)(h, h)}^{*}=J_{(f, f)(h, h)(e, e)}^{*}=J_{(h, h)(e, e)(f, f)(h, h)}^{*}$

So $(h, h)(e, e)(f, f)(h, h) \in G_{(h, h)}$, i.e.

The condition

$$
(h, h)((e, e)(f, f))^{p}(h, h) \in G_{(h, h)} \text { is right for } p=1 .
$$

According to the above assumptions $p=2$ we have

and the other hand

$$
S(h) S \subseteq S((e, e)(f, f))^{p}(h, h)(e, e)(f, f) S
$$

$$
S((e, e)(f, f))^{p}(h, h)(e, e)(f, f) S \subseteq S(h, h) S
$$

So $S(h, h) S=S((e, e)(f, f))^{p}(h, h)(e, e)(f, f) S$.

$J_{\text {Thus }}^{*}=J_{((h, h)}^{*}{ }_{((e, e)(f, f))^{p}(h, h)(e, e)(f, f)}^{*}=J_{(h, h)((e, e)(f, f))^{p+1}(h, h)}^{*}$

Whence $(h, h)((e, e)(f, f))^{p+1}(h, h) \in G_{(h, h)}$,

so by induction that

$$
(h, h)((e, e)(f, f))^{p}(h, h) \in G_{(h, h)} \text { holds. }
$$

Let $q$ be the the smallest positive integers such that $((e, e)(f, f))^{q} \in \operatorname{Re} g S$.

Then

$$
((e, e)(f, f))^{q} \in J_{(e, e)(f, f)}^{*},((e, e)(f, f))^{q} \in G_{(k, k)} \subseteq J_{(k, k)}^{*}((k, k) \in E) .
$$

Now we prove

$$
(k, k)((a, b)(x, y))^{p} \in G_{(k, k)}
$$

for every $p \geq 0$.If $p=1$. By induction that

$$
((e, e)(f, f))^{2 q} \in G_{(k, k)} \subseteq J_{(k, k)}^{*},
$$

so $\quad((e, e)(f, f))^{2 q}=((e, e)(f, f))^{2 q}(u, v)((e, e)(f, f))^{2 q}\left((u, v) \in G_{(k, k)}\right)$.

Therefor we have

$$
J_{(k, k)}^{*}=J_{(a, b))((e, e)(f, f))^{2 q}(x, y)}^{*}=J_{(x, y)(a, b)((e, e)(f, f))^{2 q}}^{*}=J_{\left.(k, k)((e, e)(f, f))^{q}(x, y)(a, b)\right)((e, e)(f, f))^{q} .}^{*}
$$

whence

$$
\begin{aligned}
& ((e, e)(f, f))^{q}(a, b)(x, y)((e, e)(f, f))^{q}= \\
& (k, k)((e, e)(f, f))^{q}(x, y)(a, b)((e, e)(f, f))^{q}(k, k) \in G_{(k, k)},
\end{aligned},
$$

by (1),(4),(5),(6) we obtain

$S((a, b)(x, y))^{r} S=S(h, h) S=S(h, h)((e, e)(f, f))^{w}(h, h) S \subseteq S((e, e)(f, f))^{q} S$;

$S((e, e)(f, f))^{q} S=S(k, k) S=S(k, k)((x, y)(a, b))^{r}(k, k) S \subseteq S((x, y)(a, b))^{r} S$.

and we consider $\mathcal{J}_{(a, b)(x, y)}^{* *}=\mathcal{J}_{(x, y)(a, b)}^{*}$; then

$$
S((a, b)(x, y))^{r} S=S((e, e)(f, f))^{q} S .
$$


Hence

$$
\mathcal{J}_{(a, b)(x, y)}^{* *}=\mathcal{J}_{(e, e)(f, f)}^{*}
$$

\section{References}

[1] LUO Xiaoqiang. The Subclasses of Characterization on $\Pi^{*}$-Regular Semigroups, Progress in Applied Mathmatics(Canadian),5(2),2013,1-5.

[2] Xiaoqiang LUO. $\Pi^{*}$-Regular Semigroups.Bulletin of Mathematical Science \&Applications (India), 1(1), (2012), 63-70.

[3] Mario Petrich and Norman R .Reilly..Completely Regular Semigroups.New York, John Wiley \& Sons ,Inc. Press, 1999.

[4] Howie J M .().An Introduction to Semigroup Theory.New York ,Academic Press, 1976.

[5] Xiaoqiang LUO. Another Property of Congruence-free Regular Semigroups.South Asian Journal of Mathematics, 2(3), 2012,238-241. 